Leandra Mara da Silva

\title{
Uma Abordagem Sensível à História para Detecção de Anomalias de Código
}

Dissertação apresentada como requisito parcial para obtenção do grau de Mestre pelo Programa de Pós-graduação em Informática do Departamento de Informática da PUC-Rio

Orientador : Prof. Carlos José Pereira de Lucena Co-Orientador: Prof. Alessandro Fabricio Garcia 


\title{
Leandra Mara da Silva
}

\section{Uma Abordagem Sensível à História para Detecção de Anomalias de Código}

Dissertação apresentada como requisito parcial para obtenção do grau de Mestre pelo Programa de Pós-graduação em Informática do Departamento de Informática do Centro Técnico Científico da PUC-Rio. Aprovada pela Comissão Examinadora abaixo assinada.

\author{
Prof. Carlos José Pereira de Lucena \\ Orientador \\ Departamento de Informática - PUC-Rio
}

Prof. Alessandro Fabricio Garcia

Co-Orientador

Departamento de Informática - PUC-Rio

Prof. Arndt von Staa

Departamento de Informática - PUC-Rio

Prof. Soeli Teresinha Fiorini

Pesquisadora - PUC-Rio

Prof. José Eugênio Leal

Coordenador Setorial do Centro Técnico Científico - PUC-Rio 
Todos os direitos reservados. É proibida a reprodução total ou parcial do trabalho sem autorização da universidade, do autor e do orientador.

\section{Leandra Mara da Silva}

Leandra Mara da Silva recebeu da Universidade Federal Fluminense (UFF) o título de bacharel em Ciência da Computação em 2007. Atualmente, participa do grupo de pesquisa OPUS que funciona junto ao Laboratório de Engenharia de Software da PUC-Rio (LES/PUC-Rio). Ela também atua como integrante do TecGraf, contribuindo no desenvolvimento do sistema v3o2 que auxilia na análise de dados sísmicos pertencentes a Petrobrás. Seus trabalhos estão relacionados a temas como manutenção de sistemas, métricas, avaliação de código, qualidade de sistemas e engenharia de software empírica.

Ficha Catalográfica

Silva, L. M.

Uma Abordagem Sensível à História para Detecção de Anomalias de Código / Leandra Mara da Silva; orientador: Carlos José Pereira de Lucena; co-orientador: Alessandro Fabricio Garcia. - Rio de Janeiro : PUC-Rio, Departamento de Informática, 2010.

v., 120 f: il. ; $29,7 \mathrm{~cm}$

1. Dissertação (mestrado) - Pontifícia Universidade Católica do Rio de Janeiro, Departamento de Informática.

Inclui referências bibliográficas.

1. Informática - Tese. 2. Manutenção de Sistemas. 3. Anomalias de Modularidade. 4. Estratégias de Detecção. 5. Métricas. 6. Métricas Sensíveis à História. 7. Engenharia de Software Empírica. I. Lucena, C. J. P.. II. Garcia, A. F.. III. Pontifícia Universidade Católica do Rio de Janeiro. Departamento de Informática. IV. Título. 
À minha família. 


\section{Agradecimentos}

Agradeço a Deus pela sua constante presença em minha vida. Agradeço pelas oportunidades de estudo, pelo conhecimento e amadurecimento adquiridos ao longo desses anos. Agradeço pelas conquistas obtidas, pelas pessoas presentes na minha vida e por este título.

Agradeço, cheia de emoção, à minha mãe, irmãs, irmão e sobrinhos. Agradeço por todo apoio e incentivo, bem como por toda admiração demonstrada a mim. Agradeço também por serem sempre tão compreensivos comigo, mesmo quando precisei ficar tantos meses sem visitá-los por conta das atividades acadêmicas.

Agradecimentos especiais a Gustavo pela maneira única que me ama e que se preocupa comigo. Também agradeço por seu apoio e presença em cada projeto que tenho assumido na minha vida e por sua paciência nos meus momentos de incerteza. Agradeço ainda por sua compreensão quando muitas vezes durante o mestrado eu não consegui lhe dar a devida atenção. Agradeço também por suas dicas e contribuições no desenvolvimento da ferramenta proposta neste trabalho.

Minhas considerações também aos amigos que constantemente me alegraram e me renovaram nos momentos de cansaço. O carinho, as brincadeiras e os sorrisos dessas pessoas sempre me trouxeram alegria. Inúmeros amigos poderiam ser citados, mas eu não teria espaço suficiente para isso. Dessa forma, como representante dos amigos de Niterói, agradeço à Luca e, como representante dos amigos de Cachoeiro, agradeço à Silvania.

Também conheci pessoas especiais na PUC, verdadeiros parceiros e companheiros de pesquisa, pessoas com quem aprendi muito. Agradeço aos colegas do LES, do Grupo de Pesquisa OPUS e do TecGraf. Agradeço à Soeli que me incentivou na realização dos primeiros estudos sobre medição de código. Ao Alessandro e ao Chico pela amizade, além de suas importantes contribuições nos trabalhos publicados. À Isela, por sua amizade e conhecimento compartilhado na área de métricas.

A profissionais como Cassino e Leonardo do TecGraf que me proporcionaram a possibilidade de aplicar os primeiros conhecimentos adquiridos sobre métricas em sistemas desenvolvidos para a Petrobrás. Ao Gustavo Carvalho, Daniele Coutinho e Vera Menezes pela atenção a mim concedida em todos os momentos em que deles precisei.

Também não poderia deixar de agradecer ao Glauco Carneiro (UFBA), Cláudio "Baiano" (UFBA) e Eduardo Figueiredo (UFMG). Por termos alcançado, juntamente com o Alessandro Garcia e o Manoel Mendonça, o prêmio de melhor artigo do CBSoft-SBES 2010. 
Agradeço também a alguns parceiros de estudo nas disciplinas cursadas. Dentre eles, Hildebrando Trannin, Kelly Leal, Katia Canepa, Elisabeth Suescun, Amadeu Barbosa, Márcio Santos, Luana Lachtermacher, Sérgio Cerqueira e outros.

Agradeço ao departamento de informática e professores, em especial à secretária Teresa e ao professor Arndt. Ao professor Carlos Lucena, meu orientador, pelo seu apoio em todas as minhas propostas de estudo.

Finalmente, ficam registradas as minhas considerações ao Alessandro, meu co-orientador. Revelo minha admiração pelo seu profissionalismo e destreza no relacionamento com seus orientados. Agradeço pela relação de confiança e amizade que estabelecemos. Agradeço por ter me concedido liberdade para crescer, mas ao mesmo tempo não ter deixado de se fazer presente nas minhas principais experiências vivenciadas durante este mestrado.

Agradeço também à CAPPES, à PUC-Rio e à Fundação Padre Leonel Franca pelo auxílio financeiro ao longo desses anos. 


\section{Resumo}

Silva, L. M.; Lucena, C. J. P.; Garcia, A. F.. Uma Abordagem Sensível à História para Detecção de Anomalias de Código. Rio de Janeiro, 2010. 120p. Dissertação de Mestrado - Departamento de Informática, Pontifícia Universidade Católica do Rio de Janeiro.

A modularização do código pode influenciar diretamente a manutenibilidade de sistemas de software. Por isso, pesquisadores têm proposto mecanismos que visam contribuir com a identificação de potenciais anomalias de modularidade no código. Nesse contexto, um mecanismo baseado em métricas que vêm sendo bastante divulgado são as estratégias de detecção. Comumente, elas são compostas por métricas que consideram apenas propriedades de versões isoladas dos sistemas. Entretanto, estudos recentes relatam que tal abordagem têm se apresentado contraproducente. Nossa pesquisa está relacionada à investigação dos possíveis benefícios de se considerar informações sobre a evolução do código na detecção de anomalias. Nesse contexto, este trabalho propõe um conjunto de métricas e de estratégias de detecção que consideram propriedades históricas do código em evolução. Além disso, uma ferramenta de medição e avaliação para dar suporte à abordagem também foi desenvolvida. Essa ferramenta permite a especificação declarativa de diferentes estratégias de detecção através de uma linguagem específica de domínio. Tal fato atende a necessidades particulares de desenvolvedores na configuração de estratégias e disponinbiliza a pesquisadores um ambiente propício à experimentação de estratégias de detecção. Ainda como contribuição desta pesquisa, é apresentada uma avaliação das estratégias em termos de precisão e revocação em dois sistemas de domínio diferentes. Resultados de estratégias convencionais e sensíveis à história são comparados em detecções de anomalias clássicas, tais como God Class, Divergent Change e Shotgun Surgery em um total de 16 versões desses sistemas. Como resultado desse estudo observou-se que a utilização de informações relacionadas à evolução do código pode trazer importantes contribuições à detecção de anomalias de código.

\section{Palavras-chave}

Manutenção de Sistemas; Anomalias de Modularidade; Estratégias de Detecção; Métricas; Métricas Sensíveis à História; Engenharia de Software Empírica; 


\section{Abstract}

Silva, L. M.; Lucena, C. J. P.; Garcia, A. F.. A History Sensitive Approach to Code Anomaly Detection. Rio de Janeiro, 2010. 120p. MsC Dissertation — Departamento de Informática, Pontifícia Universidade Católica do Rio de Janeiro.

The modularization of the code can directly influence the software maintainability. Therefore, researchers have proposed mechanisms to contribute to the identification of potential modularity anomalies in source code. In this context, a mechanism based on metrics that have been widespread are the detection strategies. Commonly, they are based on metrics that consider only properties of isolated versions of the systems. However, recent studies have reported that these strategies have been considered counter-productive. Our research is related to the investigation of the possible benefits of considering information about the code evolution to detect anomalies. In this context, this paper proposes a set of metrics and detection strategies that consider historic properties of the code evolution. Furthermore, a measuring and assessing tool to support the approach was also developed. This tool allows the declarative specification of different detection strategies through a domain-specific language. This fact meets the particular needs of developers in setting strategies and dispose to researchers an opportune environment to detection strategies experimentations. Also as a contribution of this research is presented an evaluation of strategies in terms of precision and recall in two systems of different domains. Results of conventional strategies and of history-sensitive strategies are compared in detections of classical modularity flaws, such as God Class, Divergent Change and Shotgun Surgery in a total of 16 versions of these systems. Results of this study showed that the use of information related to the code evolution can provide important contributions to detect design flaws in code.

\section{Keywords}

Software Maintenance; Code Smells; Detection Strategies; Metrics; History-Sensitive Metrics; Empirical Software Engineering; 


\section{Sumário}

1 Introdução $\quad 13$

1.1 Contextualização 13

$\begin{array}{lll}1.2 & \text { O Problema } & 14\end{array}$

$\begin{array}{lll}1.3 & \text { Limitações de Trabalhos Relacionados } & 16\end{array}$

$\begin{array}{ll}1.4 \text { Visão Geral da Solução } & 17\end{array}$

$\begin{array}{lll}1.5 & \text { Estrutura do Texto } & 20\end{array}$

2 Fundamentação $\quad 22$

2.1 Manutenção e Evolução de Sistemas 22

2.2 Refatoração e Anomalias de Modularidade de Código 25

2.3 Avaliação Sensível à História 26

3 Estado da Arte e Trabalhos Relacionados $\quad 27$

3.1 Medição de Código $\quad 27$

3.2 Estratégias de Detecção 30

3.3 Ferramentas de Suporte a Estratégias de Detecção 37

3.4 Uma técnica chamada Detecção Experta 42

3.5 Detecção Baseada em Visualização de Software 43

4 Métricas Sensíveis à História $\quad 47$

4.1 Métricas Independentes $\quad 47$

4.2 Métricas Dependentes 48

5 Estratégias de Detecção Sensíveis à História $\quad 59$

5.1 A Questão dos Valores Limites $\quad 60$

5.2 Detecções Simples 61

5.3 Detecções de Anomalias de Modularidade 63

6 Hist-Inspect: A Ferramenta de Medição e Avaliação $\quad 70$

6.1 Levantamento das Necessidades $\quad 70$

6.2 Descrição das Funcionalidades $\quad 71$

6.3 Visão Geral da Arquitetura e Funcionamento 76

7 Avaliação $\quad 79$

$\begin{array}{llr}7.1 & \text { Procedimentos } & 80\end{array}$

$\begin{array}{lll}7.2 & \text { Resultados e Discussões } & 87\end{array}$

8 Considerações Finais 101

8.1 Contribuições 102

8.2 Trabalhos Futuros 104

$\begin{array}{ll}\text { Referências Bibliográficas } & 107\end{array}$

$\begin{array}{ll}\text { A Catálogo de Métricas } & 114\end{array}$ 


\section{Lista de figuras}

3.1 Processos de filtragem e composição pertencentes a estratégias de detecção (Figura adaptada de (Marinescu 2004)).

3.2 Exemplo de God Class: classe ImageAccessor. Os valore de métricas apresentados à direita foram obtidos com base na utilização da ferramenta Together (Together 2009).

3.3 Notação para estratégia de detecção segundo (Lanza et al., 2006) 37

3.4 Ferramenta Together: suporte a estratégias de detecção 39

3.5 inCode: deteção de anomalias e sugestões de refatoração 40

3.6 iPlasma e inFusion: tela de configuração de estratégias 40

3.7 Cartão de regras da abordagem DETEX: especificação de anomalias em alto nível por meio de linguagem específica de domínio (DSL) 43

3.8 Visão de dependências entre os módulos - ferramenta Source Miner 45

3.9 Força de acoplamento entre módulos - ferramenta Source Miner 45

3.10 Visão Polimétrica - ferramenta Source Miner 46

5.1 Estratégia de detecção convencional para God Class 64

5.2 Estratégia sensível à história para God Class 65

5.3 Estratégia de detecção convencional para Shotgun Surgery 66

5.4 Estratégia sensível à história para Shotgun Surgery 67

5.5 Estratégia de detecção sensível à história para Divergent Change 68

6.1 Gráficos de evolução através da biblioteca JFreeChart 72

6.2 Apresentação de métricas sensíveis à história 73

6.3 Relatório HTML das anomalias detectadas 74

6.4 Exemplos de especificação de estratégias e respectivas anomalias $\quad 75$

6.5 Funcionamento e elementos principais da arquitetura da Hist-Inspect 77 


\section{Lista de tabelas}

3.1 Ferramentas de suporte a métricas de código 31

3.2 Comparação entre ferramentas de suporte a estratégias de detecção 42

4.1 Exemplo de dados para medição sensível à sistória 47

5.1 Exemplos de estratégias sensíveis à história com uma única métrica: estratégias simples

7.1 Principais características das aplicações do estudo 85

7.2 Detecção sensível à história no Mobile Media 88

7.3 Detecção convencional vs. sensível à história de GC no Mobile Media 90

7.4 Detecção convencional vs. sensível à história de SS no Mobile Media 92

7.5 Detecção baseada em múltiplas perspectivas visuais vs. sensível à história de GC no Mobile Media 93

7.6 Detecção baseada em múltiplas perspectivas visuais vs. sensível à história de DC no Mobile Media 94

7.7 Detecção convencional vs. sensível à história de GC no Health Watcher 96

7.8 Detecção sensível à história de DC no Health Watcher 96

B.1 Oráculos para o Mobile Media. Anomalias de classes: God Class (GC), Divergent Change (DC) e Shotgun Surgery (SS). Células em tons de cinza indicam que o módulo não existe nas versões especificadas ou que existe com um outro nome (refatoração "rename").

B.2 Oráculo para o Mobile Media. Anomalia de método: Feature Envy (FE).Células em tons de cinza indicam que o módulo não existe nas versões especificadas ou que existe com um outro nome (refatoração "rename").

B.3 Oráculos para o Health Watcher. Anomalias de classes: God Class (GC) e Divergent Change (DC). Células em tons de cinza indicam que o módulo não existe nas versões especificadas ou que existe com um outro nome (refatoração "rename"). 